\title{
Dynamical analysis for a vector-like dark energy
}

\author{
Ricardo C. G. Landim (i) ${ }^{\mathrm{a}}$ \\ Departamento de Física-Matemática, Instituto de Física, Universidade de São Paulo, Rua do Matão, Travessa R, 187, \\ Cidade Universitária, 05508-090 São Paulo, SP, Brazil
}

Received: 11 May 2016 / Accepted: 19 August 2016 / Published online: 31 August 2016

(c) The Author(s) 2016. This article is published with open access at Springerlink.com

\begin{abstract}
In this paper we perform a dynamical analysis for a vector field as a candidate for the dark energy, in the presence of a barotropic fluid. The vector is one component of the so-called cosmic triad, which is a set of three identical copies of an abelian field pointing mutually in orthogonal directions. In order to generalize the analysis, we also assumed the interaction between dark energy and the barotropic fluid, with a phenomenological coupling. Both matter and dark energy eras can be successfully described by the critical points, indicating that the dynamical system theory is a viable tool to analyze asymptotic states of such cosmological models.
\end{abstract}

\section{Introduction}

Around $95 \%$ of the universe today corresponds to two kinds of components whose nature is still unknown. The first one, called dark energy, is believed to be responsible for the current accelerated expansion of the universe $[1,2]$ and it is dominant at present times $(\sim 68 \%)$ [3]. In addition to ordinary matter, the remaining $27 \%$ of the energy content of the universe is a form of matter that interacts in principle only gravitationally, known as dark matter. The simplest dark-energy candidate is the cosmological constant, whose equation of state $w_{\Lambda}=p_{\Lambda} / \rho_{\Lambda}=-1$ is in agreement with the Planck results [3]. This attempt, however, suffers from the so-called cosmological constant problem, a huge discrepancy of 120 orders of magnitude between the theoretical prediction and the observed data. Such a huge disparity motivates physicists to look into more sophisticated models. This can be done either looking for a deeper understanding of where the cosmological constant comes from, if one wants to derive it from first principles, or considering other possibilities for accelerated expansion. In the former case, an attempt is the famous KKLT model [4], and in the latter one, possibilities are even

\footnotetext{
a e-mail: rlandim@if.usp.br
}

broader, with modifications of general relativity, additional matter fields and so on (see [5-7] and references therein). Moreover, the theoretical origin of this constant is still an open question, with several attempts but with no definitive answer yet.

Among a wide range of alternatives, the field theory can provide some other candidates. The simplest one is the canonical scalar field [8-12], although non-canonical scalar fields have also been explored (tachyon field [13,14], k-essence [15], or supergravity-inspired models [16-19], for instance). Another dark-energy candidate is a spin-1 particle, described by a vector field. To be consistent with the homogeneity and isotropy of the universe, there should be three identical copies of the vector field, which one with the same magnitude and pointing mutually in the orthogonal direction. They are called cosmic triad and were proposed in [20]. Other possibilities of vector dark energy are shown in [21-26].

In addition, the two components of the dark sector may interact with each other [7,27-40] (see [41] for a recent review), since their densities are comparable and the interaction can eventually alleviate the coincidence problem [42,43].

When the dark-energy candidate is in the presence of a barotropic fluid (with an equation of state $w_{m}=p_{m} / \rho_{m}$ ) the relevant evolution equations can be converted into an autonomous system and the asymptotic states of the cosmological models can be analyzed. Such approach was done for uncoupled dark energy (quintessence, tachyon field and phantom field for instance [44-49]) and coupled dark energy [28,34,50-55], but it remained to be done for a vector-like dark energy, whose interesting properties were explored in [20]. In this paper, we use the linear dynamical systems theory to investigate the critical points that come from the evolution equations for the vector-like dark energy, considering also the possibility of interaction between the two components of the dark sector, where we propose a phenomenological coupling. The fixed points found can successfully describe the matter-dominated universe and the current stage 
of accelerated expansion, provided that the interaction is sufficiently small.

The rest of the paper is organized as follows. In Sect. 2 we present the basics of the interacting dark energy and the dynamical analysis theory. Section 3 contains the dynamics of the vector-like dark energy in the light of the dynamical system theory, where the critical points and their stabilities are presented. Section 4 is reserved for our conclusions. We use Planck units ( $\left.\hbar=c=1=M_{p l}=1\right)$ throughout the text.

\section{Interacting dark energy and the dynamical system theory}

As a generalization of the continuity equation, we consider an interaction between the dark energy, described by the cosmic triad, and a barotropic fluid, in such a way that the total energy-momentum tensor is still conserved. Dark energy has an energy density $\rho_{A}$ and pressure $p_{A}$, with an equation of state given by $w_{A}=p_{A} / \rho_{A}$. In the flat FriedmannLemaître-Robertson-Walker (FLRW) background with a scale factor $a \equiv a(t)$, the continuity equations are

$\dot{\rho}_{A}+3 H\left(\rho_{A}+p_{A}\right)=-\mathscr{Q}$,

$\dot{\rho_{m}}+3 H\left(1+w_{m}\right) \rho_{m}=\mathscr{Q}$,

respectively, where $H=\dot{a} / a$ is the Hubble rate, $\mathscr{Q}$ is the coupling, and the dot is a derivative with respect to the cosmic time $t$. The index $m$ stands for the barotropic fluid, with $w_{m}=0$ for non-relativistic matter and $w_{m}=1 / 3$ for radiation. The case of $\mathscr{Q}>0$ corresponds to a dark-energy transformation into the barotropic fluid, while $\mathscr{Q}<0$ is the transformation in the opposite direction. In principle, the coupling can depend on several variables $\mathscr{Q}=\mathscr{Q}\left(\rho_{m}, \rho_{A}, \ldots\right)$, so that, inspired by the quintessence case [27,28], where the coupling is $Q \rho_{m} \dot{\phi}$, we assume the phenomenological interaction $\mathscr{Q}=3 Q \rho_{m} \dot{A} / a$, where $Q$ is a positive constant. The coupling has this form in order for the right-hand side of the Proca-like Eq. (9) to be no longer zero but to equal $Q \rho_{m} \cdot{ }^{1}$ The case with negative $Q$ is similar and we will not consider it here because the minus sign of the case $\mathscr{Q}<0$ can be absorbed into $\dot{A}$, instead of considering $Q<0$.

To deal with the dynamics of the system, we define dimensionless variables. The new variables are going to characterize a system of differential equations in the form

$X^{\prime}=f[X]$,

\footnotetext{
${ }^{1}$ In the scalar field case the coupling $Q \rho_{m} \dot{\phi}$ leads to the equation of motion in the FLRW background which also equals $Q \rho_{m}$.
}

where $X$ is a column vector of dimensionless variables and the prime is the derivative with respect to $\log a$, where we set the present scale factor $a_{0}$ to be one. The critical points $X_{c}$ are those ones that satisfy $X^{\prime}=0$. In order to study stability of the fixed points, we consider linear perturbations $U$ around them, thus $X=X_{c}+U$. At the critical point the perturbations $U$ satisfy the following equation:

$U^{\prime}=\mathscr{J} U$

where $\mathscr{J}$ is the Jacobian matrix. The stability around the fixed points depends on the nature of the eigenvalues $(\mu)$ of $\mathscr{J}$, in such a way that they are stable points if they all have negative values, unstable points if they all have positive values and saddle points if at least one eigenvalue has positive (or negative) value, while the other ones have opposite sign. In addition, if any eigenvalue is a complex number, the fixed point can be stable $(\operatorname{Re} \mu<0)$ or unstable $(\operatorname{Re} \mu>0)$ spiral, due to the oscillatory behavior of its imaginary part.

\section{Vector-like dark-energy dynamics}

The Lagrangian for three identical copies of an abelian field (called cosmic triad in [20]), here uncoupled to matter, is given by

$\mathscr{L}_{A}=-\sqrt{-g} \sum_{a=1}^{3}\left(\frac{1}{4} F^{a \mu \nu} F_{\mu \nu}^{a}+V\left(A^{a 2}\right)\right)$,

where $F_{\mu \nu}^{a}=\partial_{\mu} A_{\nu}^{a}-\partial_{\nu} A_{\mu}^{a}$ and $V\left(A^{2}\right)$ is the potential for the vector field, which breaks gauge invariance, with $A^{a 2} \equiv A_{\mu}^{a} A^{a \mu}$. The energy-momentum tensor of the field is obtained varying the Lagrangian (5) with respect to the metric and it is $T_{\mu \nu}^{A}=\sum_{a=1}^{3} T_{\mu \nu}^{a}$, where

$T_{\mu \nu}^{a}=\left[F_{\mu \rho}^{a} F_{\nu}^{a \rho}+2 \frac{\mathrm{d} V}{\mathrm{~d} A^{a 2}} A_{\mu}^{a} A_{\nu}^{a}-g_{\mu \nu}\left(\frac{1}{4} F_{\rho \sigma}^{a \rho \sigma}+V\left(A^{2}\right)\right)\right]$.

Varying (5) with respect to the fields $A_{\mu}^{a}$ gives the equations of motion

$\partial_{\mu}\left(\sqrt{-g} F^{a \mu \nu}\right)=2 \sqrt{-g} V^{\prime} A^{a v}$,

where from now on we use $V^{\prime} \equiv \frac{\mathrm{d} V}{\mathrm{~d} A^{a 2}}$.

In an expanding universe, with FLRW metric and scale factor $a$, each one of the three vectors should be along a coordinate axis with same magnitude. An ansatz for the $i$ components of the vector $A_{\mu}^{a}$ compatible with homogeneity and isotropy is 
$A_{i}^{a}=\delta_{i}^{a} A(t)$,

where a scalar product with an unit vector is implicit. From (7) the component $A_{0}^{a}$ is zero and using (8) into (7) the equation of motion becomes

$\ddot{A}+H \dot{A}+2 V^{\prime} A=0$.

The pressure and energy density for the cosmic triad is obtained from (6)

$\rho_{A}=\frac{3 \dot{A}^{2}}{2 a^{2}}+3 V$,

$p_{A}=\frac{\dot{A}^{2}}{2 a^{2}}-3 V+2 V^{\prime} \frac{A^{2}}{a^{2}}$.

With this ansatz $z^{2}$ the potential depends now on $V\left(3 A^{2} / a^{2}\right)$ and the prime is the derivative with respect to $3 A^{2} / a^{2}$. We assume that the potential is given by $V=V_{0} e^{-\frac{3 \lambda A^{2}}{a^{2}}}$, where $V_{0}$ is a constant. With this form the quantity $-V^{\prime} / V$ will be constant, as we will see soon. Thus, for the comoving vector $A_{i c}^{a}=A_{i}^{a} \cdot a$ (as used in [20]), the potential does not have an explicit dependence on the scale factor. If the cosmic triad were massless, we would have $\dot{A} \propto a^{-1}$, thus $\rho_{A} \propto a^{-4}$, as it should be for relativistic matter.

As we have said, we assume the interaction between the cosmic triad with a barotropic fluid given by $3 Q \rho_{m} \dot{A} / a$, thus the right-hand side of Eq. (9) becomes $Q \rho_{m} a$. In the presence of a barotropic fluid, the Friedmann equations are

$$
\begin{aligned}
& H^{2}=\frac{1}{3}\left(\frac{3 \dot{A}^{2}}{2 a^{2}}+3 V+\rho_{m}\right), \\
& \dot{H}=-\frac{1}{2}\left(\frac{2 \dot{A}^{2}}{a^{2}}+2 V^{\prime} \frac{A^{2}}{a^{2}}+\left(1+w_{m}\right) \rho_{m}\right) .
\end{aligned}
$$

We now proceed to the dynamical analysis of the system.

\subsection{Autonomous system}

The dimensionless variables are defined as

$$
\begin{aligned}
x & \equiv \frac{\dot{A}}{\sqrt{2} H a}, \quad y \equiv \frac{\sqrt{V(\phi)}}{H}, \quad z \equiv \frac{A}{a}, \\
\lambda & \equiv-\frac{V^{\prime}}{V}, \quad \Gamma \equiv \frac{V V^{\prime \prime}}{V^{\prime 2}} .
\end{aligned}
$$

\footnotetext{
${ }^{2}$ In [20] the author used a comoving vector ansatz: $A_{\mu}^{a}=\delta_{\mu}^{a} A(t) \cdot a$. This choice leads, of course, to a different equation of motion, energy density, and pressure. However, the effect due to the scale factor that here appears in the denominator of $\rho_{A}$ and $p_{A}$, for instance, appears as a Hubble friction term $(H \dot{A})$ in the same expressions.
}

The dark-energy density parameter is written in terms of these new variables as

$\Omega_{A} \equiv \frac{\rho_{A}}{3 H^{2}}=x^{2}+y^{2}$,

so that Eq. (12) can be written as

$\Omega_{A}+\Omega_{m}=1$

where the density parameter of the barotropic fluid is defined by $\Omega_{m}=\rho_{m} /\left(3 H^{2}\right)$. From Eqs. (15) and (16) $x$ and $y$ are restricted in the phase plane by the relation

$0 \leq x^{2}+y^{2} \leq 1$

due to $0 \leq \Omega_{A} \leq 1$.

The equation of state $w_{A}=p_{A} / \rho_{A}$ becomes

$w_{A}=\frac{x^{2}-3 y^{2}-2 \lambda z^{2}}{3 x^{2}+3 y^{2}}$.

Depending on the value of $\lambda$ the equation of state can be less than minus one.

The total effective equation of state is

$$
\begin{aligned}
w_{\text {eff }}= & \frac{p_{A}+p_{m}}{\rho_{A}+\rho_{m}}=w_{m}+x^{2}\left(\frac{1}{3}-w_{m}\right)-y^{2}\left(1+w_{m}\right) \\
& -\frac{2}{3} \lambda y^{2} z^{2}
\end{aligned}
$$

with an accelerated expansion for $w_{\text {eff }}<-1 / 3$. The dynamical system for the variables $x, y, z$ and $\lambda$ are

$$
\begin{aligned}
\frac{\mathrm{d} x}{\mathrm{~d} N}= & -x+\sqrt{2} y^{2} z \lambda-\frac{3}{\sqrt{2}} Q\left(1-x^{2}-y^{2}\right) \\
& -x\left[y z^{2} \lambda-x^{2}+y^{2}-\frac{1+3 w_{m}}{2}\left(1-x^{2}-y^{2}\right)\right],
\end{aligned}
$$

$$
\begin{aligned}
\frac{\mathrm{d} y}{\mathrm{~d} N}= & -3 y z \lambda(\sqrt{2} x-z) \\
& -y\left[y^{2} z^{2} \lambda-2 x^{2}-\frac{3}{2}\left(1+w_{m}\right)\left(1-x^{2}-y^{2}\right)\right],
\end{aligned}
$$

$\frac{\mathrm{d} z}{\mathrm{~d} N}=2 x-z$

$\frac{\mathrm{d} \lambda}{\mathrm{d} N}=-6 \lambda^{2} z(\Gamma-1)(\sqrt{2} x-z)$.

\subsection{Critical points}

The fixed points of the system are obtained by setting $\mathrm{d} x / \mathrm{d} N=0, \mathrm{~d} y / \mathrm{d} N=0, \mathrm{~d} z / \mathrm{d} N=0$, and $\mathrm{d} \lambda / \mathrm{d} N=0$ 
Table 1 Critical points $(x, y$, and $z$ ) of Eq. (20) for the vector-like dark energy. The table shows the correspondent equation of state for the dark energy (18), the effective equation of state (19), the density parameter for dark energy (15) and the eigenvalues of the Jacobian matrix in Eq. (4)

\begin{tabular}{llllllllll}
\hline Point & $x$ & $y$ & $z$ & $w_{A}$ & $\Omega_{A}$ & $w_{\text {eff }}$ & $\mu_{1}$ & $\mu_{2}$ & $\mu_{3}$ \\
\hline (a) & \pm 1 & 0 & \pm 2 & $\frac{1}{3}(1-8 \lambda)$ & 1 & $\frac{1}{3}$ & -1 & $1 \pm 3\left(\sqrt{2} Q-w_{m}\right)$ & $2+6(2-\sqrt{2}) \lambda$ \\
(b) & $\frac{\sqrt{2} Q}{w_{m}-1 / 3}$ & 0 & $2 x$ & $\frac{1}{3}(1-8 \lambda)$ & $\frac{2 Q^{2}}{\left(w_{m}-1 / 3\right)^{2}}$ & $6 Q^{2}$ & -1 & $-\frac{1}{2}+9 Q^{2}$ & $\frac{3}{2}+Q^{2}(9+108(2-\sqrt{2}) \lambda)$ \\
(c) & 0 & 1 & 0 & -1 & 1 & -1 & $-3\left(1+w_{m}\right)$ & $-\frac{3}{2}(1+\sqrt{1+8 \sqrt{2} \lambda})$ & $-\frac{3}{2}(1-\sqrt{1+8 \sqrt{2} \lambda})$ \\
\hline
\end{tabular}
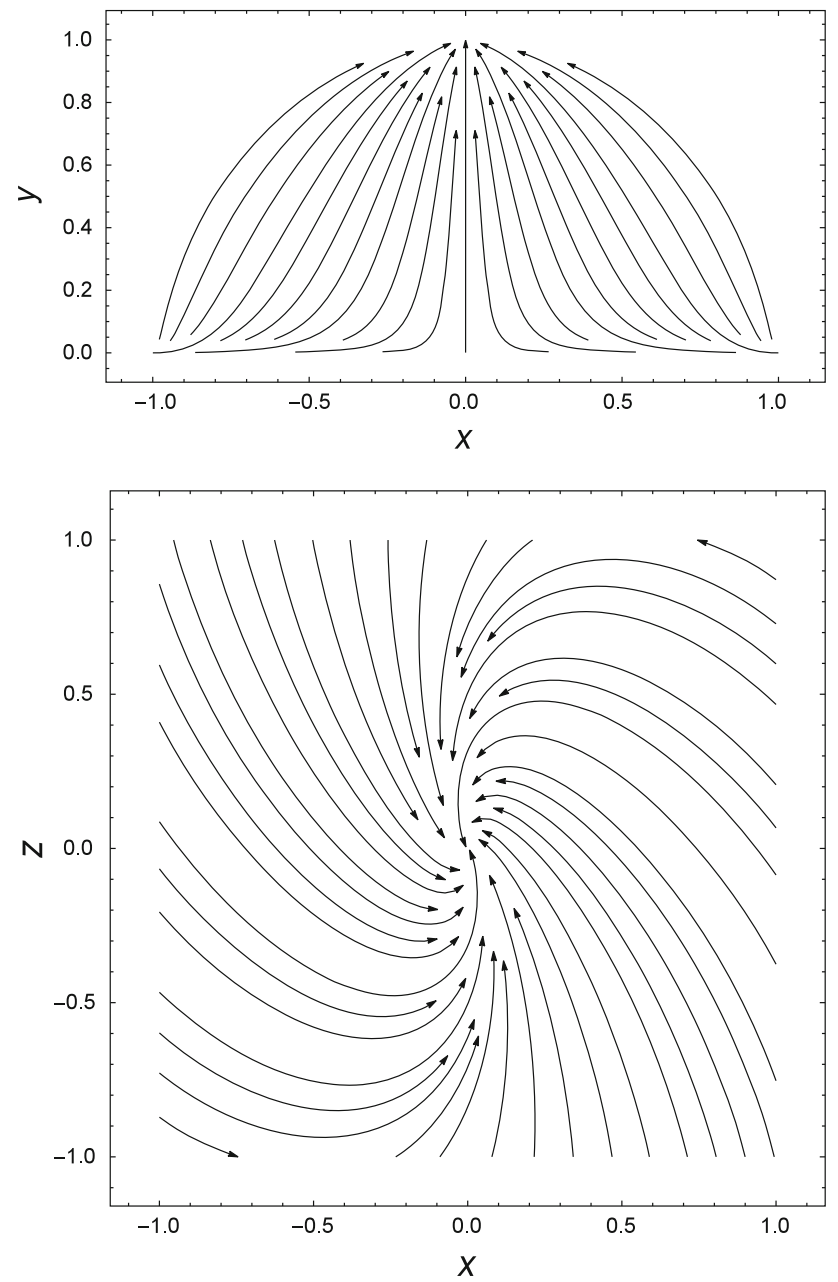

Fig. 1 Phase portrait of the system, with $Q=0$ and $\lambda=-0.3$. All trajectories converge to the attractor (c) at $x=0, y=1$, and $z=$ 0 , which is a stable spiral that describes the dark-energy-dominated universe. The top panel shows the slice $z=0$, while the bottom panel shows the phase plane at $y=1$

in Eqs. (20)-(23). When $\Gamma=1, \lambda$ is constant the potential is $V\left(A^{2}\right)=V_{0} e^{\frac{-3 \lambda A^{2}}{a^{2}}} \cdot 3$ Different from the scalar field case, where $V=V_{0} e^{-\lambda \phi}$, the exponent of the potential also

\footnotetext{
$\overline{3}$ The equation for $\lambda$ is also equal zero when $z=0$ or $\lambda=0$, so that $\lambda$ should not necessarily be constant, for the fixed point with this value of $z$. However, for the case of dynamical $\lambda$, the correspondent eigenvalue is equal to zero, indicating that the fixed points are not hyperbolic.
}

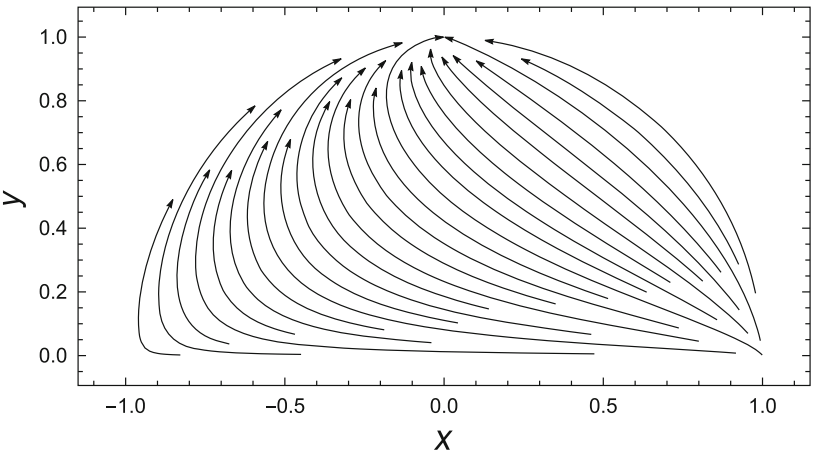

Fig. 2 Phase portrait of the system, with $Q=1 / \sqrt{6}$ and $\lambda=-0.3$. All trajectories converge to the attractor (c) at $x=0, y=1$, and $z=0$, which is a stable spiral that describes the dark-energy dominated universe. The panel shows only the slice $z=0$ because the phase plane for $y=1$ is similar to that one showed in Fig. 1

depends on the scale factor $a$. The fixed points are shown in Table 1 with the eigenvalues of the Jacobian matrix. Notice that $y$ cannot be negative.

The point (a) corresponds to a radiation solution, once $w_{\text {eff }}=1 / 3$. It can be a saddle or a stable point, depending on the value of $Q$ and $\lambda$. However, the universe is dominated by the cosmic triad, as indicated by $\Omega_{A}=1$, and therefore the fixed point does not describe a radiation-dominated universe, since $\Omega_{m}=0$. The point (b) is valid only for $w_{m} \neq 1 / 3$ and it is a saddle point, since two eigenvalues are negative and one is positive. Since $y=0$ for this critical point, $x^{2}$ should be less than or equal to one (since $\Omega_{A} \leq 1$ ), so the coupling should be $Q \leq 1 / \sqrt{2}$. However, this critical point can describe a matter-dominated universe only if $Q=0$ or sufficiently small $Q \ll 1$, so that $w_{\text {eff }} \approx 0$, as so for $\Omega_{A}$. The last fixed point (c) is an attractor and describes a dark-energy dominated universe $\left(\Omega_{A}=1\right)$ that leads to an accelerated expansion of the universe, since $w_{A}=w_{\text {eff }}=-1$. It is a stable spiral if $\lambda<-1 /(8 \sqrt{2})$, otherwise it is a saddle point. The potential for this condition for $\lambda$ is $V=V_{0} e^{3|\lambda| z^{2}}$ and it behaves as the cosmological constant at the fixed point, since $z \equiv A / a=0$ for (c). Once the coupling is constant and sufficiently small (to the fixed point (b) describe the matterdominated universe), it has the same value, of course, for the point (c). 


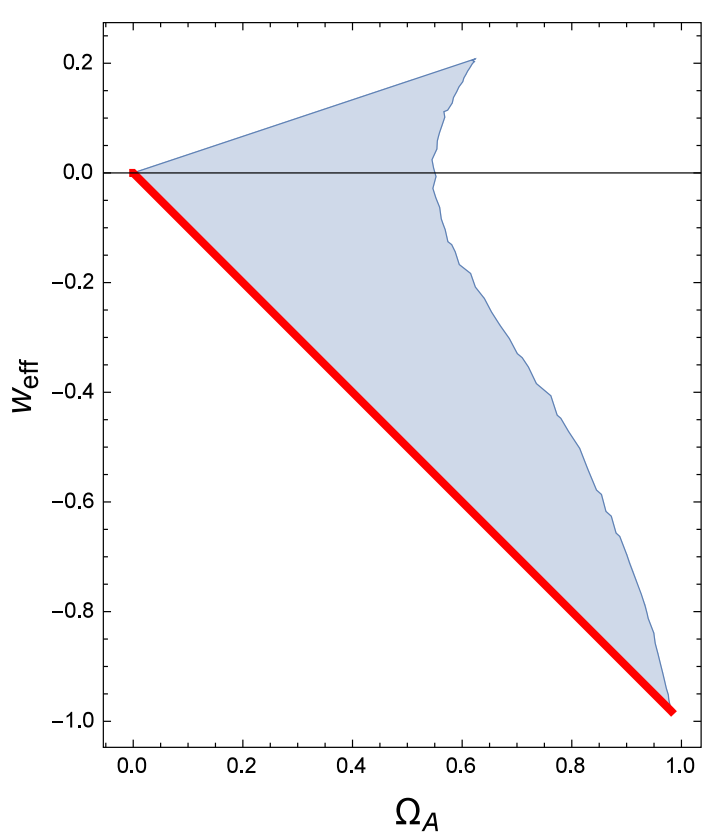

Fig. 3 Effective equation of state $w_{\text {eff }}(19)$ as a function of the darkenergy density parameter $\Omega_{A}$ (15). This parametric plot is independent of $Q$, once both $w_{\text {eff }}$ and $\Omega_{A}$ have no explicit dependence on the interaction. The blue shaded region represents the allowed values of $w_{\text {eff }}$ and $\Omega_{A}$. We used $w_{m}=0$ since this is the only allowed value for the fixed point (b). The red line shows the transition from the fixed point (b) $\left(\Omega_{A}=0\right)$ to the fixed point (c) $\left(\Omega_{A}=1\right)$

We show the phase portrait of the system in Figs. 1 $(Q=0)$ and $2(Q=1 / \sqrt{6})$. The latter case is shown just to illustrate how the interaction affects the phase portrait, although we expect a very small $Q$, as discussed for the fixed point (b). We see that all trajectories converge to the attractor point.

In Fig. 3 we show the effective equation of state $w_{\text {eff }}(19)$ as a function of the dark-energy density parameter $\Omega_{A}(15)$, where the blue shaded region represents the allowed values of $w_{\text {eff }}$ and $\Omega_{A}$. The red line shows the transition from the fixed point (b) $\left(\Omega_{A}=0\right)$ to the fixed point (c) $\left(\Omega_{A}=1\right)$.

\section{Conclusions}

In this paper we used the dynamical system theory to investigate if a vector-like dark energy, similar to [20], in the presence of a barotropic fluid can lead to the three cosmological eras, namely, radiation, matter, and dark energy. The analysis was generalized for the case of coupled dark energy, with a phenomenological interaction $3 Q \dot{A} \rho_{m} / a$. There are fixed points that successfully describe the matter-dominated and the dark-energy-dominated universe. Only the radiation era was not cosmologically viable, however, if one is interested in the last two periods of the evolution of the universe, the dynamical system theory provides a good tool to analyze asymptotic states of such cosmological models.

Acknowledgments This work is supported by FAPESP Grant No. 2013/10242-1.

Open Access This article is distributed under the terms of the Creative Commons Attribution 4.0 International License (http://creativecomm ons.org/licenses/by/4.0/), which permits unrestricted use, distribution, and reproduction in any medium, provided you give appropriate credit to the original author(s) and the source, provide a link to the Creative Commons license, and indicate if changes were made. Funded by $\mathrm{SCOAP}^{3}$.

\section{References}

1. A.G. Riess et al., Observational evidence from supernovae for an accelerating universe and a cosmological constant. Astron. J. 116, 1009-1038 (1998)

2. S. Perlmutter et al., Measurements of Omega and Lambda from 42 high redshift supernovae. Astrophys. J. 517, 565-586 (1999)

3. P.A.R. Ade et al., Planck 2013 results. XVI. Cosmological parameters. Astron. Astrophys. 571, A16 (2014)

4. S. Kachru, R. Kallosh, A. Linde, S.P. Trivedi, de sitter vacua in string theory. Phys. Rev. D 68, 046005 (2003)

5. E.J. Copeland, M. Sami, S. Tsujikawa, Dynamics of dark energy. Int. J. Mod. Phys. D 15, 1753-1936 (2006)

6. G. Dvali, G. Gabadadze, M. Porrati, 4D gravity on a brane in 5D Minkowski space. Phys. Lett. B 485, 208 (2000)

7. S. Yin, B. Wang, E. Abdalla, C. Lin, Transition of equation of state of effective dark energy in the Dvali-Gabadadze-Porrati model with bulk contents. Phys. Rev. D 76, 124026 (2007)

8. P.J.E. Peebles, B. Ratra, Cosmology with a time variable cosmological constant. Astrophys. J. 325, L17 (1988)

9. B. Ratra, P.J.E. Peebles, Cosmological consequences of a rolling homogeneous scalar field. Phys. Rev. D 37, 3406 (1988)

10. J.A. Frieman, C.T. Hill, R. Watkins, Late time cosmological phase transitions. 1. Particle physics models and cosmic evolution. Phys. Rev. D 46, 1226-1238 (1992)

11. J.A. Frieman, C.T. Hill, A. Stebbins, I. Waga, Cosmology with ultralight pseudo Nambu-Goldstone bosons. Phys. Rev. Lett. 75, 2077 (1995)

12. R.R. Caldwell, R. Dave, P.J. Steinhardt, Cosmological imprint of an energy component with general equation of state. Phys. Rev. Lett. 80, 1582 (1998)

13. T. Padmanabhan, Accelerated expansion of the universe driven by tachyonic matter. Phys. Rev. D 66, 021301 (2002)

14. J.S. Bagla, H.K. Jassal, T. Padmanabhan, Cosmology with tachyon field as dark energy. Phys. Rev. D 67, 063504 (2003)

15. C. Armendariz-Picon, V.F. Mukhanov, P.J. Steinhardt, A dynamical solution to the problem of a small cosmological constant and late time cosmic acceleration. Phys. Rev. Lett. 85, 4438-4441 (2000)

16. P. Brax, J. Martin, Quintessence and supergravity. Phys. Lett. B468, 40-45 (1999)

17. E.J. Copeland, N.J. Nunes, F. Rosati, Quintessence models in supergravity. Phys. Rev. D 62, 123503 (2000)

18. R.C.G. Landim, Cosmological tracking solution and the SuperHiggs mechanism. Eur. Phys. J. C 76(8), 430 (2016)

19. R.C.G. Landim, Holographic dark energy from minimal supergravity. Int. J. Mod. Phys. D 25(4), 1650050 (2016)

20. C. Armendariz-Picon, Could dark energy be vector-like? JCAP 0407, 007 (2004) 
21. T. Koivisto, D.F. Mota, Vector field models of inflation and dark energy. JCAP 0808, 021 (2008)

22. K. Bamba, S.D. Odintsov, Inflation and late-time cosmic acceleration in non-minimal Maxwell- $F(R)$ gravity and the generation of large-scale magnetic fields. JCAP 0804, 024 (2008)

23. V. Emelyanov, F.R. Klinkhamer, Possible solution to the main cosmological constant problem. Phys. Rev. D 85, 103508 (2012)

24. V. Emelyanov, F.R. Klinkhamer, Reconsidering a higher-spin-field solution to the main cosmological constant problem. Phys. Rev. D 85, $063522(2012)$

25. V. Emelyanov, F.R. Klinkhamer, Vector-field model with compensated cosmological constant and radiation-dominated FRW phase. Int. J. Mod. Phys. D 21, 1250025 (2012)

26. S. Kouwn, P. Oh, C.-G. Park, Massive photon and dark energy. Phys. Rev. D 93(8), 083012 (2016)

27. C. Wetterich, The Cosmon model for an asymptotically vanishing time dependent cosmological 'constant'. Astron. Astrophys. 301, 321-328 (1995)

28. L. Amendola, Coupled quintessence. Phys. Rev. D 62, 043511 (2000)

29. G.R. Farrar, P.J.E. Peebles, Interacting dark matter and dark energy. Astrophys. J. 604, 1-11 (2004)

30. Z.-K. Guo, Y.-Z. Zhang, Interacting phantom energy. Phys. Rev. D. 71, 023501 (2005)

31. R.-G. Cai, A. Wang, Cosmology with interaction between phantom dark energy and dark matter and the coincidence problem. JCAP 0503, 002 (2005)

32. Z.-K. Guo, R.-G. Cai, Y.-Z. Zhang, Cosmological evolution of interacting phantom energy with dark matter. JCAP 0505, 002 (2005)

33. X.-J. Bi, B. Feng, H. Li, X. Zhang, Cosmological evolution of interacting dark energy models with mass varying neutrinos. Phys. Rev. D. 72, 123523 (2005)

34. B. Gumjudpai, T. Naskar, M. Sami, S. Tsujikawa, Coupled dark energy: towards a general description of the dynamics. JCAP 0506, 007 (2005)

35. B. Wang, Y.-G. Gong, E. Abdalla, Transition of the dark energy equation of state in an interacting holographic dark energy model. Phys. Lett. B 624, 141-146 (2005)

36. B. Wang, Y. Gong, E. Abdalla, Thermodynamics of an accelerated expanding universe. Phys. Rev. D 74, 083520 (2006)

37. B. Wang, C.-Y. Lin, E. Abdalla, Constraints on the interacting holographic dark energy model. Phys. Lett. B 637, 357-361 (2006)

38. B. Wang, C.-Y. Lin, D. Pavon, E. Abdalla, Thermodynamical description of the interaction between dark energy and dark matter. Phys. Lett. B 662, 1-6 (2008)
39. S. Micheletti, E. Abdalla, B. Wang, A field theory model for dark matter and dark energy in interaction. Phys. Rev. D 79, 123506 (2009)

40. A.A. Costa, L.C. Olivari, E. Abdalla, Quintessence with Yukawa interaction. Phys. Rev. D 92(10), 103501 (2015)

41. B. Wang, E. Abdalla, F. Atrio-Barandela, D. Pavon. Dark matter and dark energy interactions: theoretical challenges, cosmological implications and observational signatures. Rept. Prog. Phys. 79(9), 096901 (2016)

42. W. Zimdahl, D. Pavon, Interacting quintessence. Phys. Lett. B521, 133-138 (2001)

43. L.P. Chimento, A.S. Jakubi, D. Pavon, W. Zimdahl, Interacting quintessence solution to the coincidence problem. Phys. Rev. D 67, 083513 (2003)

44. E.J. Copeland, A.R. Liddle, D. Wands, Exponential potentials and cosmological scaling solutions. Phys. Rev. D 57, 4686-4690 (1998)

45. S.C.C. Ng, N.J. Nunes, F. Rosati, Applications of scalar attractor solutions to cosmology. Phys. Rev. D 64, 083510 (2001)

46. E.J. Copeland, M.R. Garousi, M. Sami, S. Tsujikawa, What is needed of a tachyon if it is to be the dark energy? Phys. Rev. D 71, 043003 (2005)

47. X.-H. Zhai, Y.-B. Zhao, A cosmological model with complex scalar field. Nuovo Cim. B 120, 1007-1016 (2005)

48. J. De-Santiago, J.L. Cervantes-Cota, D. Wands, Cosmological phase space analysis of the $\mathrm{F}(\mathrm{X})-\mathrm{V}(\phi)$ scalar field and bouncing solutions. Phys. Rev. D 87(2), 023502 (2013)

49. J. Dutta, W. Khyllep, N. Tamanini, Cosmological dynamics of scalar fields with kinetic corrections: beyond the exponential potential. Phys. Rev. D 93(6), 063004 (2016)

50. S. Tsujikawa, General analytic formulae for attractor solutions of scalar-field dark energy models and their multi-field generalizations. Phys. Rev. D 73, 103504 (2006)

51. L. Amendola, M. Quartin, S. Tsujikawa, I. Waga, Challenges for scaling cosmologies. Phys. Rev. D 74, 023525 (2006)

52. X.-M. Chen, Y.-G. Gong, E.N. Saridakis, Phase-space analysis of interacting phantom cosmology. JCAP 0904, 001 (2009)

53. R.C.G. Landim, Coupled tachyonic dark energy: a dynamical analysis. Int. J. Mod. Phys. D 24, 1550085 (2015)

54. R.C.G. Landim, Coupled dark energy: a dynamical analysis with complex scalar field. Eur. Phys. J. C 76(1), 31 (2016)

55. N. Mahata, S. Chakraborty. Dynamical system analysis for DBI dark energy interacting with dark matter. Mod. Phys. Lett. A 30(02), 1550009 (2015) 